\title{
COMPARISON OF INTEGRATION ALGORITHMS FOR REAL-TIME HYBRID SIMULATION USING FREQUENCY DOMAIN-BASED ERROR INDICATORS
}

\author{
Reza Mirza Hessabi ${ }^{1, *}$, Ali Ashasi-Sorkhabi ${ }^{2, *}$, Yanhui Liu ${ }^{3, \dagger}$, and Oya Mercan ${ }^{4, *}$ \\ * Department of Civil Engineering, University of Toronto, Toronto, ON, Canada \\ 1 e-mail: reza.hessabi@mail.utoronto.ca \\ 2 e-mail: ali.ashasisorkhabi@mail.utoronto.ca \\ ${ }^{4}$ oya.mercan@utoronto.ca
}

${ }^{\dagger}$ State Key Laboratory for Seismic Reduction/Control \& Structural Safety, Guangzhou University, China ${ }^{3}$ e-mail: liuyanhui@gzhu.edu.cn

Keywords: Real-time hybrid simulation, frequency domain-based error indicators, integration algorithms.

\begin{abstract}
Real-time hybrid simulation (RTHS) is a testing method that combines computer simulation (i.e., analytical substructure) with physical testing (i.e., experimental substructure). This enables investigation of dynamic characteristics of load-rate dependent complex structures. To ensure reliable experimental results, even in the presence of sophisticated controllers and compensation methods, it is necessary to evaluate the accuracy of the success of the hydraulic actuators in imposing the command displacements. With recent developments in tracking error monitoring, new indicators have been proposed that are based on frequency domain analysis and can successfully uncouple phase (lag and lead) and amplitude (overshoot and undershoot) errors and quantify them. These new frequency-based error indicators are not structure-specific and can be reliably used to examine the effectiveness of each component of RTHS inner (i.e., servo-control) and outer (i.e., integration algorithm) loops. In this paper, frequency domain-based (FDB) error indicators are employed to evaluate the effectiveness of five commonly-used integration algorithms (i.e. central difference method, explicit Newmark method, discrete state space formulation with a zero-order hold and first-order hold, the state-space formulation by Zhang et al., and the alpha method) in different test scenarios. The applicability of the FDB error indicators to nonlinear systems is first verified through numerical simulations performed on a nonlinear system with predefined parameters. Then these indicators are used to post-process the experimental results obtained from RTHS's with various integration algorithms. The experiments are carried out on a system with a nonlinear analytical substructure and a linear experimental substructure at the University of Toronto. Findings from numerical simulation and experimental results demonstrate that the FDB method is an efficient approach to compare performances of different integration algorithms.
\end{abstract}




\section{INTRODUCTION}

Real-time hybrid simulation (RTHS) is a powerful experimental technique to investigate the dynamic response of complex and rate-dependent structures. In this method, there is an integration algorithm that solves the equation of motion and resulting displacements are imposed on a physical test specimen by using hydraulic actuators. The restoring force and displacement information from the deformed specimen are measured and then used to compute next step command displacements. The block diagram of a typical RTHS is shown in Fig. 1.

OUTER LOOP

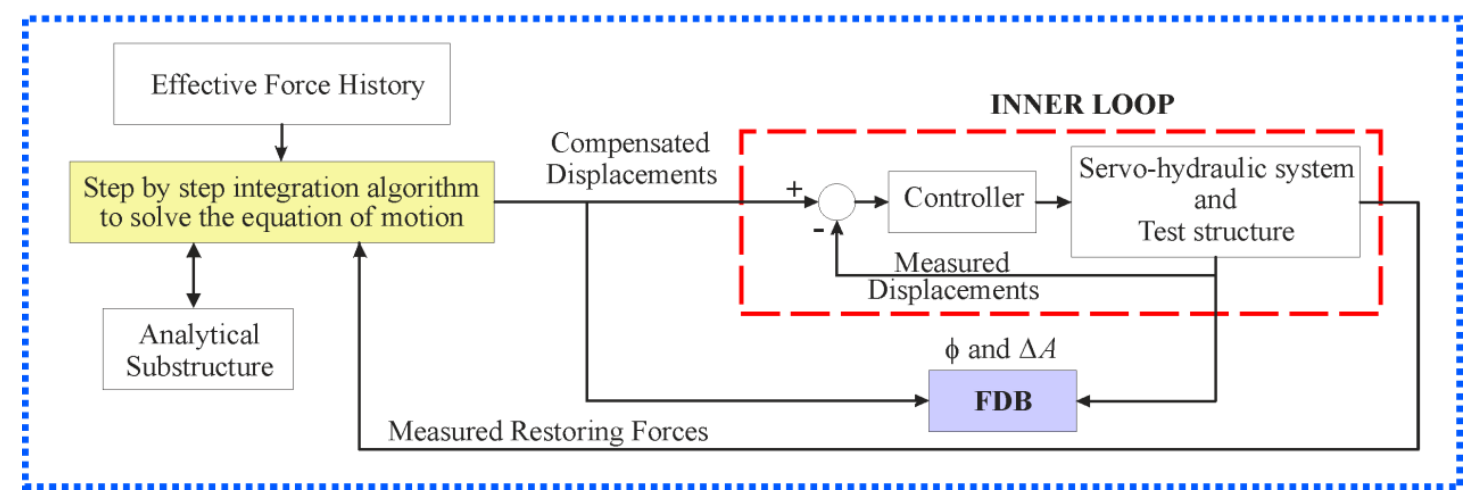

Figure 1: Block diagram of the inner and outer loops of RTHS.

In the process of generating the command displacements, integration algorithms are central and are used to solve the discretized equations of motion of structures. In order for an integration algorithm to be reliable it must be stable and accurate. Several implicit and explicit integration methods such as central difference methods, explicit Newmark method have been used extensively in the past [1,2] and many other robust integration algorithms have been recently developed for RTHS. The analysis of integration algorithms are typically carried out either in the time domain or frequency domain. List of some of the available criteria for investigating the performance of integration algorithms are listed in [3]. One of the studies on the performance evaluation of integration algorithms was carried out by Mugan in which he found that frequency domain analysis can describe all of the time domain properties of an integration algorithm [4]. A frequency domain analysis will also be used in this paper to evaluate different integration algorithms.

In addition to the accuracy of the integration algorithms, another key factor for obtaining reliable RTHS results is system errors. Errors in a hybrid simulation can be attributed to either the numerical components or the experimental components, and even in the steps required to couple the two together. Due to the vulnerability of RTHS to error propagation, which can affect the accuracy and even the stability of the entire experiment, it is imperative to estimate and monitor the errors. Current available error assessment measures are categorized in [5, 6] and can also be divided into time domain and frequency domain assessment measures. In this paper, the application of one of the most recently developed frequency domain error assessment measures is proposed to evaluate the performance of various integration algorithms.

\section{FREQUENCY DOMAIN-BASED ERROR INDICATORS}

\subsection{Background}

Since the introduction of the RTHS, there has been a continuous effort on development and implementation of proper methods to assess the success of the simulation results. Current 
methods for estimation of local response error such as the tracking error (i.e., the difference between the command and measured displacements) usually use time domain properties [7], cumulative growth of energy errors [8], synchronization subspace plots [9, 10] or the frequency domain response of the signals [11] to develop error monitors and indicators.

Although the abovementioned indicators are useful in evaluating the quality of the test results and have been successfully used by other researchers, there was a need for a new indicator that was not structure or test specific and was capable of uncoupling the phase and amplitude errors. Mirza Hessabi et al. [12] introduced a new set of error indicators (i.e., Frequency Domain-Based (FDB) error indicators) to solve these problems. Moreover, unlike their predecessors, the implementation of these new indicators is not computationally expensive and therefore they can be executed in realtime and serve as online indicators.

The FDB error indicators are developed using discrete Fourier transform (DFT). These indicators can be employed to find the corresponding values of the amplitude ratio and phase error within each moving time window. As shown in Eq. 1, the amplitude ratio is defined in terms of the ratio of the command over measured displacement amplitudes.

$$
\Delta A=A_{c} / A_{m}
$$

Similarly, a phase error is defined in Eq. 2 based on the discrepancy of the phase shifts between the command and measured signals. Positive and negative phase error values from Eq. 2 show phase lead and lag, respectively. Similarly, a value greater than one obtained from Eq. 1 is an undershoot error and a value less than one represents overshoot.

$$
\phi=\phi_{c}-\phi_{m}
$$

It should be emphasized that the closer $\Delta A$ and $\phi$ values are to 1 and 0 , respectively, the more accurate is the actuator control and the more reliable are the RTHS results.

In order to determine the FDB error indicators, first the DFT power spectra of the command and measured signals are computed independently and then, from each power spectrum, the frequency that corresponds to the largest amplitude is located (i.e. $\bar{f}_{c}$ and $\bar{f}_{m}$ for command and measured signals, respectively) [12]. As it is shown in Eq. 3, the ratio between the magnitudes of the power spectra at the frequencies of $\bar{f}_{c}$ and $\bar{f}_{m}$ can be used to determine the amplitude ratio,

$$
\Delta A=\left|U_{c}\left(\overline{f_{c}}\right)\right| /\left|U_{m}\left(\bar{f}_{m}\right)\right|
$$

Likewise, the phase error defined in Eq. 4 is found as the difference of the DFT phase spectrum values of the two signals at $\bar{f}_{c}$ and $\bar{f}_{m}$.

$$
\phi=\phi_{c}^{*}\left(\bar{f}_{c}\right)-\phi_{m}^{*}\left(\bar{f}_{m}\right)
$$

\subsection{Application of FDB error indicators to nonlinear substructures}

Although several numerical simulations were performed in [12] to illustrate the advantages of using the FDB error indicators in comparison to other available error assessment measures, all of the evaluated cases were limited to structural elements with linear behaviour. In order to verify the applicability of the FDB error indicators to nonlinear systems, in this section Simulink is used and numerical simulations are carried out for a test structure that shows a hysteretic behavior as shown in Fig. 2-b. The hysteretic model exhibits elasto-plastic behaviour with strain hardening (the post-yield stiffness is $10 \%$ of the initial stiffness). Also, the yield force of the model is $25 \%$ of the weight of the analytical structure. The mass and damping ratio of the considered test structure are 1 ton and $5 \%$, respectively. In addition, a natural period of vibration of 0.7 seconds is assumed for the test structure and the initial stiffness is determined 
accordingly. The Simulink models for modeling the servo-hydraulic set-up are the same as [13] and a sinusoidal signal with the amplitude of $0.2 \mathrm{~m}$ and frequency of $0.8 \mathrm{~Hz}$ is chosen as the input. Finally, the PID controller for the simulation is tuned with the $k_{p}, k_{i}$ and $k_{d}$ coefficients of 25, 1 and 1, respectively. The measured and command displacements for the described system is shown in Fig. 2-a. As it can be seen, the measured displacements have smaller amplitudes (undershoot) and are lagging behind the command signals.
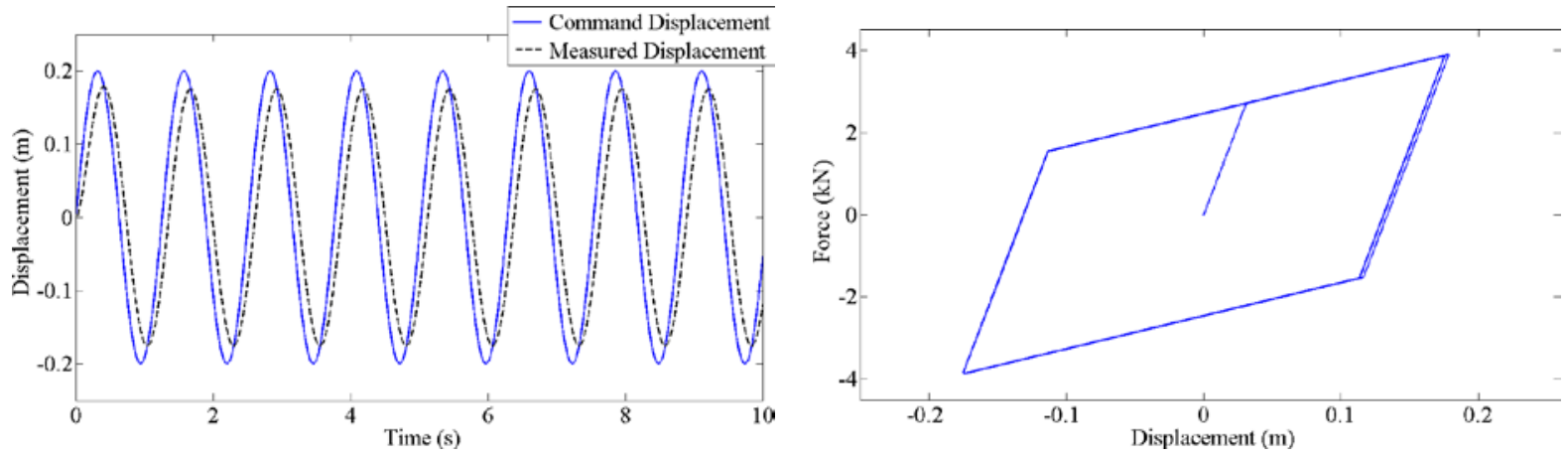

Figure 2: a) Command and measured displacements in time domain, and b) hysteresis loop for the structure with nonlinearity.

In Fig. 3, FDB error indicators are employed to measure the tracking error. The oscillation of the indicators around the mean values is due to the application of moving windows and Fourier transforms in the calculation of these indicators. The average values of FDB amplitude ratio and phase error are 1.160 and -28.189 degrees, respectively.
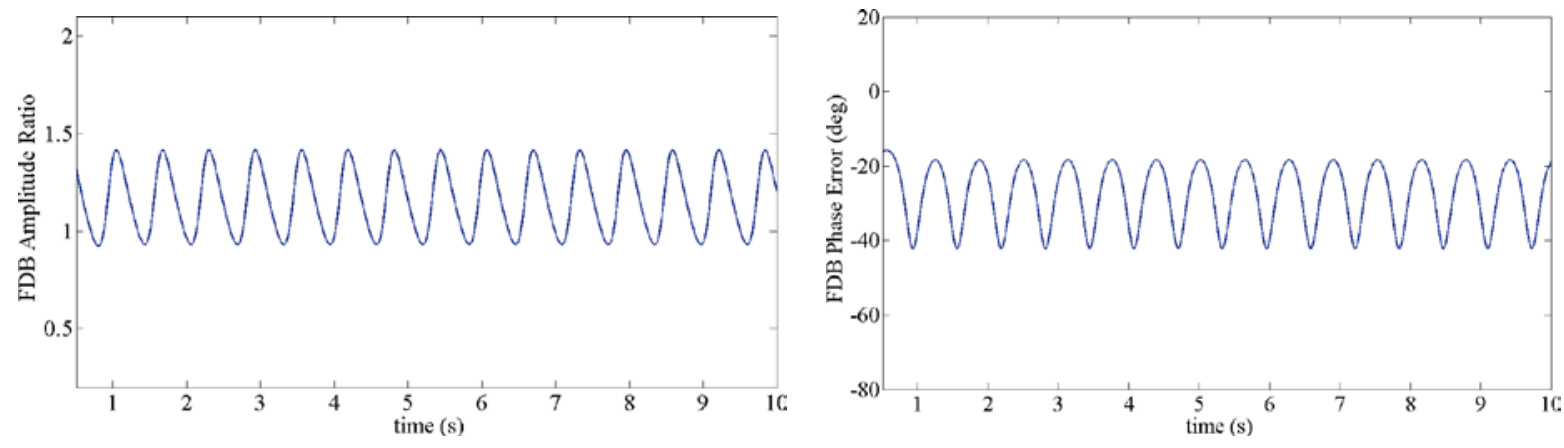

Figure 3: FDB error indicators for the structure with nonlinearity.

In order to verify these values and illustrate the accuracy of the FDB indicators, the time domain plots of command and measured displacements in Fig. 2-a, are enlarged around the $4^{\text {th }}$ and $5^{\text {th }}$ seconds in Fig. 4.
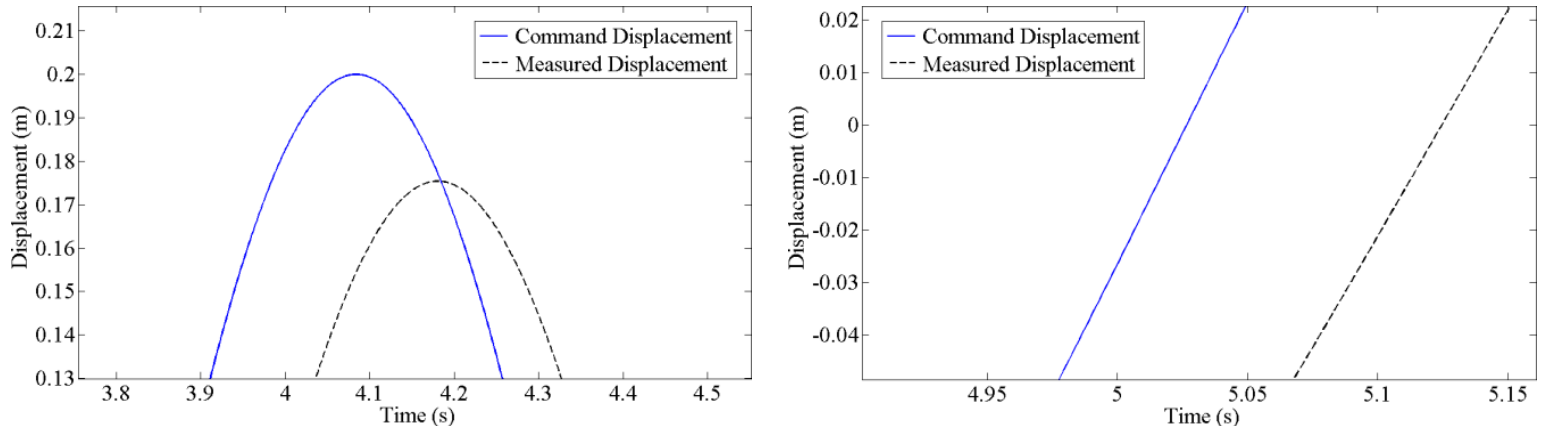

Figure 4: Enlarged time windows around a) $4^{\text {th }}$ and b) $5^{\text {th }}$ seconds to verify FDB error indicator results. 
The enlarged view around the $4^{\text {th }}$ second (Fig. 4-a) shows that the peak command displacement maximum (i.e., $0.2 \mathrm{~m})$ is 1.140 times larger than the measured displacement $(0.175$ $\mathrm{m})$. Also, from a closer look at Fig. 4-b it can be observed that the measured displacement is delayed by 96 time steps. Considering the size of the time steps, (i.e., 1/1024 sec) this translates to a phase lag of 26.857 degrees. These observations are in agreement with the average FDB error indicators from Fig. 3.

\section{PERFORMANCE EVALUATION OF THE INTEGRATION ALGORITHMS USING FDB ERROR INDICATORS}

In this study, error analysis is not discussed from a mathematical point of view. Rather, FDB error indicators are employed to examine the experimental RTHS results and compare the accuracy of the integration methods with each other. A series of RTHS are carried out for which everything except the integration algorithms is kept identical. This way, the difference in the tracking performance of the simulations can be attributed to the effects of integration algorithms on the accuracy of the simulations. The RTHS were conducted for a system that was comprised of a linear spring with the stiffness of $194 \mathrm{~N} / \mathrm{mm}$ as the experimental substructure and a nonlinear analytical substructure in which Bouc-Wen model was used to model hysteretic type nonlinearity. The integration methods that are considered in this section were the central difference method [1], explicit Newark method [2], discrete state space formulation with a zero-order hold and first-order hold [14], the state-space formulation by Zhang et al [15] and the Hilber-Hughes-Taylor (HHT) (also called asalpha method) [16]. The system was subjected to the Canoga Park record from the 1994 Northridge earthquake. Fig. 5 shows the measured displacements for one of the RTHS with the central difference integration algorithm.

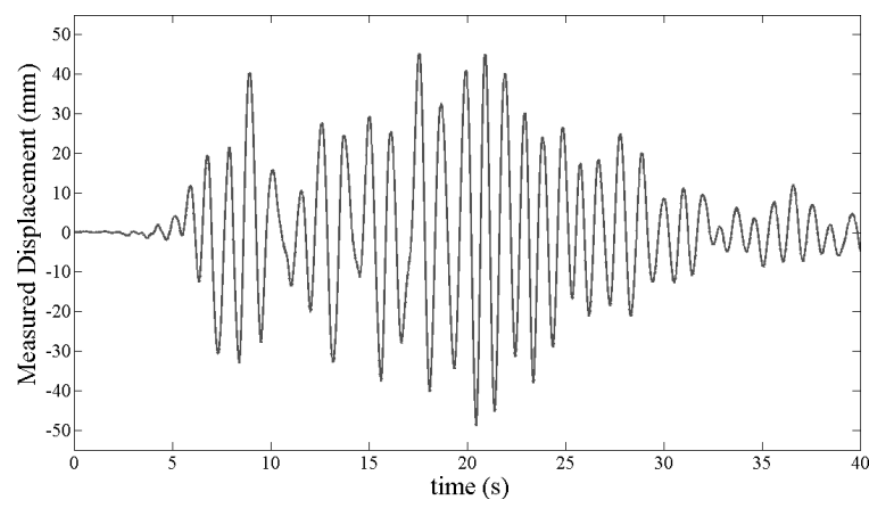

Figure 5: Measured displacement time history for the RTHS with central difference integration algorithm.

Similar to Section 2.2., FDB error indicators are used to measure the errors in each simulation. As a sample, the results for the test with the central difference method are shown below. 

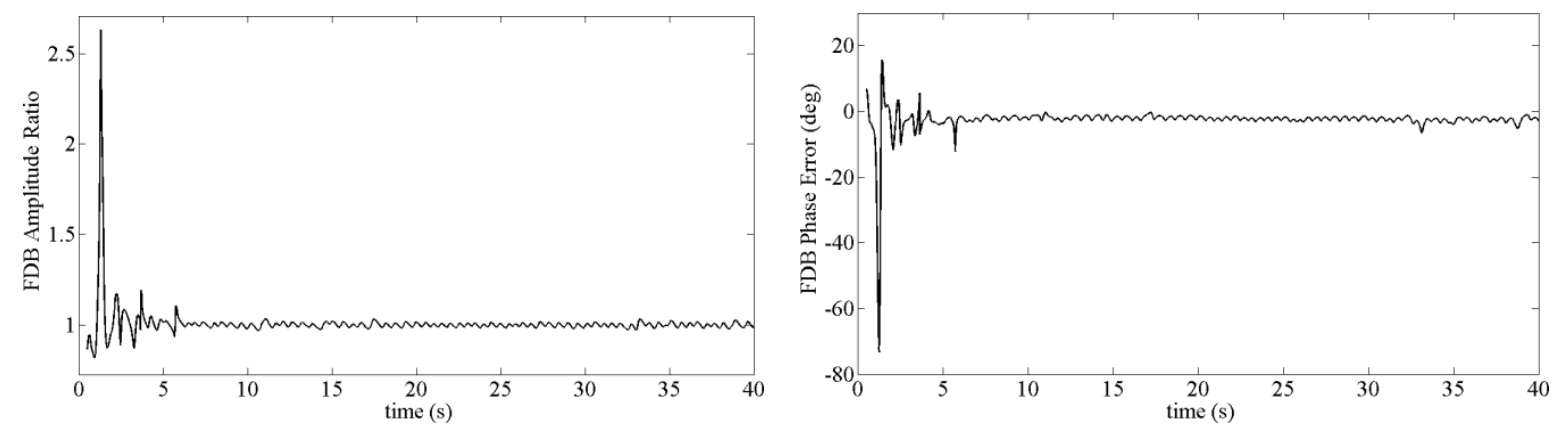

Figure 6: FDB error indicators for the RTHS with central difference integration algorithm.

As it can be seen from Fig. 6 maximum variation in the FDB error indicators occur in the first five seconds of the simulation where the command displacements are small. After the initial five seconds, the amplitude ratio oscillates around one (no amplitude error condition) and the phase error oscillate around -2.5 degrees (phase lag). It should be emphasized again that the closer amplitude ratio and phase error values are to 1 and 0 , respectively, the more reliable are the RTHS results. Since the error indicator values for different integration algorithms are relatively close, enlarged views of these indicators are shown in Fig. 7. It can be observed that the application of the central difference method leads to the highest amplitude ratios. The worst results (i.e., the highest phase errors with sudden changes) belong to the simulations with the explicit Newmark and discrete state space formulation with a zero-order hold integration algorithms.
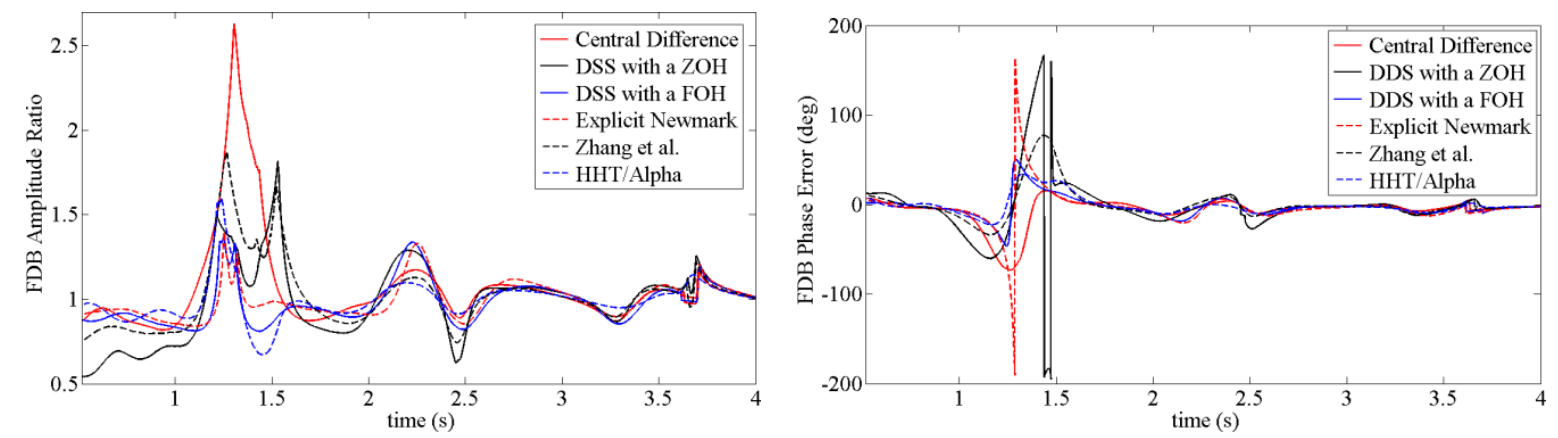

Figure 7: Enlarged views of the FDB error indicators for the RTHS with different integration algorithms.

Finally, in order to compare the abovementioned algorithms numerically, mean values of the FDB error indicators are calculated and reported in Table 1. To have other means to assess the accuracy of the experiment results, the maximum tracking error (MTE) and root mean square (RMS) of the tracking error are also included in Table 1.

\begin{tabular}{lcccc}
\hline Integration Algorithm & MTE & RMS & $\begin{array}{c}\text { Amplitude } \\
\text { Ratio }\end{array}$ & $\begin{array}{c}\text { Phase Error } \\
\text { (deg) }\end{array}$ \\
\hline Central Difference Method & 2.063 & 0.590 & 1.006 & -2.679 \\
Explicit Newmark Method & 2.093 & 0.606 & 0.999 & -2.529 \\
DDS with a zero-order hold & 2.069 & 0.600 & 1.001 & -2.534 \\
DDS with a first-order hold & 2.116 & 0.597 & 0.998 & -2.416 \\
Zhang et al. method & 2.045 & 0.583 & 1.003 & -2.157 \\
HHT/Alpha method & 2.031 & 0.620 & 0.999 & -2.230 \\
\hline
\end{tabular}

Table 1: Error assessment measures for RTHS with various integration algorithms. 
Table 1 shows that the averaged values fail to fully capture the effects of large local errors. Thus FDB error indicators can be successfully used as an evaluation tool to monitor the performance of RTHS during the entire simulation.

\section{CONCLUSIONS}

This paper presents a general approach utilizing the FDB error indicators to evaluate the different integration algorithms on the performance of RTHS. The state-of-the-art for error assessment measures is reviewed briefly and the formulation of the FDB error indicators is explained. Then a numerical case study is introduced where a system with a bilinear behaviour is used and the applicability of the FDB error indicators to nonlinear systems is verified. Followed by the numerical simulations, six different integration algorithms are selected to experimentally demonstrate the effectiveness of the FDB error indicators in characterizing the effects of selected integration methods. Based on the assumptions and limitations of this paper, it can be concluded that the effects of an integration algorithms on the performance of the RTHS cannot be judged based on averaged measures. Instead an evaluation tool is needed that can be used to monitor the effects throughout the simulation. FDB error indicators can be successfully used to achieve this goal and can assist in the selection of a suitable integration algorithm.

\section{REFERENCES}

[1] A.K. Chopra, Dynamics of Structures, 4th Edition. Prentice Hall: Upper Saddle River, New Jersey, 2011.

[2] N.M. Newmark, A method of computation for structural dynamics. Journal of Engineering Mechanics, 85, 67-94, 1959.

[3] C. Chen, J.M. Ricles, Development of direct integration algorithms for structural dynamics using discrete control theory. Journal of Engineering Mechanics, 134, 676-683, 2008.

[4] A. Mugan, Discrete equivalent time integration methods for transient analysis. International Journal for Numerical Methods in Engineering, 57, 2043-2075, 2003.

[5] R. Christenson, S. Dyke, J. Zhang, G. Mosqueda, C. Chen, N. Nakata, P. Laplace, W. Song, Y. Chae, G.A. Marshall, G. Ou, C.A. Riascos Gonzales, C. Song, Hybrid simulation: a discussion of current assessment measures. https://nees.org/resources/12876, 2014.

[6] R. Mirza Hessabi, O. Mercan, Assessment measures for hybrid simulation: a discussion of recent developments. https://nees.org/resources/13483, 2015.

[7] G. Mosqueda, B. Stojadinovic, S.A. Mahin, Realtime error monitoring for hybrid simulation. Part I: methodology and experimental verification. Journal of Structural Engineering, 133, 1100-1108, 2007.

[8] R. Mirza Hessabi, O. Mercan, A practical approach to the phase and amplitude error estimation of pseudodynamic testing. Structures Congress, American Society of Civil Engineers (ASCE), Pittsburgh, PA, May 2-4, 2013.

[9] O. Mercan, J.M. Ricles, Experimental studies on realtime testing of structures with elastomeric dampers. Journal of Structural Engineering, 135, 1124-1133, 2009. 
[10] R. Mirza Hessabi, O. Mercan, Phase and amplitude error indices for error quantification in pseudodynamic testing. Earthquake Engineering and Structural Dynamics, 41, 13471364, 2012.

[11] T. Guo, C. Chen, W. Xu, F. Sanchez, A frequency response analysis approach for quantitative assessment of actuator tracking for real-time hybrid simulation. Smart Materials and Structures, 23, 045042, 2014.

[12] R. Mirza Hessabi, A. Ashasi-Sorkhabi, O. Mercan, A new tracking error-based adaptive controller for servo-hydraulic actuator control. Journal of Vibration and Control, 1077546314548205, 2014. DOI: 10.1177/1077546314548205.

[13] H. Moosavi, R. Mirza Hessabi, O. Mercan, Numerical simulation and analysis of nonlinear state - space control design for hydraulic actuator control. Structural Control and Health Monitoring, 2015. DOI: 10.1002/stc.1731.

[14] G.F. Franklin, J.D. Powell, M. Workman, Digital control of dynamic systems, 3rd Edition. Addison Wesley Longman, Menlo Park, 1997.

[15] Y.F. Zhang, R. Sause, J.M. Ricles, C.J. Naito, Modified predictor-corrector numerical scheme for real-time pseudo dynamic tests using state-space formulation. Earthquake Engineering and Structural Dynamics, 34, 271-288, 2005.

[16] H.M. Hilber, T.J.R. Hughes, R.L. Taylor, Improved numerical dissipation for time integration algorithms in structural dynamics. Earthquake Engineering and Structural Dynamics, 5, 283-292, 1977. 